Correspondence: W-M Chan

Hong Kong Eye Hospital

$147 \mathrm{~K}$ Argyle Street Kowloon

Hong Kong

Tel: + 85226322879

Fax: + 85226482943

E-mail: cwm6373@netvigator.com

Eye (2005) 19, 825-827. doi:10.1038/sj.eye.6701662;

published online 3 September 2004

Sir,

\section{Total hyphema following postoperative enoxaparin} (Clexane)

Postoperative hyphema is a well-known complication of intraocular surgery. Internists and ophthalmologists are uncertain of the significance of antiplatelet drugs and systemic anticoagulation therapy side effects in these patients. Controversies exist regarding the safety of these drugs with regard to risks of ocular haemorrhage. We report what we believe to be the first case of total hyphema in a patient prescribed enoxaparin (Clexane Aventis Pharma Pty Ltd) in the postoperative period who had undergone a redo trabeculectomy.

\section{Case report}

A 73-year-old man underwent a redo trabeculectomy enhanced with 5-fluorouracil (5FU). The surgery was uncomplicated. The bleb was massaged on postoperative day 2 with no hyphaema seen following this. There had been no intraocular bleeding with previous surgery. The patient's general medical history included aortic and mitral valve replacement with long-term warfarin therapy. The haematocrit was 0.425 (normal $0.40-0.55$ ) and platelet count being $2.03 \times 10^{5} / \mathrm{cm}^{-3}$ (normal 1.5$4.5 \times 10^{5}$ ). Warfarin was not stopped prior to surgery. The INR on the day of the surgery was 1.6. At 2 days following the surgery INR was found to be 1.3. Enoxaparin $1 \mathrm{mg} / \mathrm{kg}(80 \mathrm{mg})$ to cover the risk of emboli from the prosthetic valves was recommended by the physician. On day 4 postoperatively the IOP was $12 \mathrm{mmHg}$ with a functioning filtration bleb, clear cornea and noninflamed anterior chamber. However, that night he suffered a total hyphaema with pressures rising to $64 \mathrm{mmHg}$. The patient denied rubbing his eyes. Medical therapy partially controlled the pressure. A paracentesis was only partially successful and aspiration of the clot with vitrectomy cutter was performed. This controlled the IOP. The residual blood behind the iris and around the intraocular lens gradually resorbed over the next 3 months. The final outcome of the surgery was an IOP of $12 \mathrm{mmHg}$, with cornea stained and decompensated and a vision of counting fingers at 12 months.

\section{Comment}

Most hyphaemas following intraocular surgery occur at the time or in the immediate postoperative period. ${ }^{1}$ The source of the bleeding appears to be the iris root or scleral incision.

Crystalline warfarin sodium is an anticoagulant that acts by inhibiting vitamin K-dependant coagulation factors including Factors II (prothrombin), VII, IX and X (intrinsic pathway). Enoxaparin, a low molecular weight heparin (MW $4500 \mathrm{Da}$ ), not only inhibits thrombin directly, but also acts on prothrombinase complex, that is, Factors $X a$ and Va calcium and phospholipid (extrinsic pathway) and by releasing an endothelium-bound pool of tissue factor inhibitor (TFPI). The drugs act on the different limbs of the Coagulation Cascade and intersect at Factor X. Warfarin takes 5-7 days for peak activity. Enoxaparin takes 3-12 h for peak activity to start, has greater bioavailability, and a longer, often unpredictable half-life. . $^{2,3}$

In hyphaemas a fibrin-platelet clot forms that reaches maximum stabilisation by day $5-7$. These clots do not show any fibroblastic activity unlike other parts of the body and finally break down via the fibrinolytic system. ${ }^{4}$

We propose that enoxaparin with its unpredictable and prolonged half-life acting via the extrinsic arm of the coagulation cascade tipped the balance in favour of haemorrhage when the clot was remodelling at day 4 . The fall in INR in 2 days after surgery we feel is probably due to the preoperative antibiotic cover with systemic ampicillin/gentamicin and the midazolam used as part of anaesthetic. ${ }^{2}$

The physicians were concerned with the inadequate anticoagulation in a patient with two prosthetic heart valves. As the effects of warfarin may take up to 4 days to occur, a decision was made to add the low molecular weight heparin similar to what is done when initiating warfarin therapy although this is usually with normal intravenous heparin.

The incidence of sight-threatening complications in patients who have undergone intraocular surgery while receiving long-term anticoagulation therapy with warfarin is not significantly different from patients who were not on such treatment. ${ }^{5,6}$ Furthermore, no differences in major haemorrhagic events are noted among patients who had their anticoagulation treatment stopped in the perioperative period for ocular surgery when compared to patients who did not stop therapy. 7,8 
This case illustrates the challenges of operating on patients requiring anticoagulation therapy. The introduction of the low molecular weight heparin agents has led to the promise of easier administration. However, the consequence is the unpredictable half-life and the difficulty in monitoring their effects. We would recommend using caution when prescribing a low molecular weight heparin agent in the postoperative period especially in someone already using warfarin. There is a good temporal relationship between the addition of enoxaparin and the total hyphema; however, this is at present an isolated finding and therefore could have occurred by chance.

\section{References}

1 Albert DM, Jacobiec FA. Principles and Practice of Ophthalmology. B Saunders: London, 1994, pp 2516-2520.

2 Braunwaldt E, Fauci AS. Harrison's Principles of Internal Medicine, 15th edition. McGraw-Hill Publishing: Newyork, 2001, pp 354-360.

3 Buckley MM, Sorkin EM. Enoxaparin: a review of its pharmacology and clinical applications in the prevention and treatment of thromboembolic disorders. Drugs 1992; 44: 465-497.

4 Sankar PS, Chen TC, Grosskreutz CL. Traumatic hyphema. Int Ophthalmol Clin 2002; 42(3): 57-68.

5 McCormack P, Simcock PR, Tulb AB. Management of anticoagulated patient for ophthalmic surgery. Eye 1993; 7: 749-750.
6 Gainey SP, Robertson DM, Fay N, Ilstrup D. Ocular surgery on patients receiving long term warfarin therapy. $A m \mathrm{~J}$ Ophthalmol 1989; 108: 142-146.

7 Katz J, Feldman MA, Bass EB, Lubomski LH, Tielsch JM, Petty BG. Risks and benefits of anticoagulant and antiplatelet medication use before cataract surgery. Ophthalmology 2003; 110(9): 1784-1788.

8 Koehler MP, Sholiton DB. Spontaneous hyphema resulting from warfarin. Ann Ophthalmol 1983; 15: 858-859.

S Sharan', G Painter² and JR Grigg ${ }^{1}$

${ }^{1}$ Department of Ophthalmology

Save Sight Institute, University of Sydney

Macquarie Street, Sydney, Australia

${ }^{2}$ Gordon Eye Surgery

802-808 Pacific Highway

Gordon 2072, Sydney, Australia

Correspondence: 5 Sharan

Tel: +61293827300

Fax: +61293827372

E-mail: sapnasd@yahoo.co.uk

Eye (2005) 19, 827-828. doi:10.1038/sj.eye.6701664; published online 3 September 2004 